\title{
Androgen Regulates the Expression and Function of Connexin 43 in Granulosa Cells via the PKC Signalling Pathway
}

\section{Yangyang Zhang}

Peking University First Hospital

Yang Xu ( $\nabla$ xuyangm@126.com )

Peking University First Hospital

\section{Yanrong Kuai}

Peking University First Hospital

\section{Sheng Wang}

Peking University First Hospital

Jiao Yu

Peking University First Hospital

\section{Research}

Keywords: androgen, testosterone, connexin 43, phosphorylation Cx43, PKC, poor ovarian response

Posted Date: September 22nd, 2021

DOl: https://doi.org/10.21203/rs.3.rs-795972/v2

License: (c) (1) This work is licensed under a Creative Commons Attribution 4.0 International License. Read Full License 


\section{Abstract}

Background: Poor ovarian response (POR) is one of the most challenging issues in assisted reproductive technology. Given the importance of androgens in follicle recruitment, growth and development, domestic and foreign reproductive centers have added androgen as an adjuvant to in vitro fertilization protocols for patients with POR to improve the ovarian response. The mechanism underlying the effects of androgens is unclear. The aim of this research was to study the effects of testosterone on granulosa cells and the underlying mechanisms.

Methods: The human ovarian granulosa cell line KGN was treated with testosterone at various concentrations, an androgen receptor antagonist (flutamide) and a protein kinase $C$ (PKC) inhibitor (GF109203X). The protein levels of PKC and phosphorylated connexin 43 ( $p-C x 43$ ) were measured using Western blot analysis. The location and distribution of $\mathrm{Cx} 43$ and $\mathrm{p}-\mathrm{Cx} 43$ were determined by immunofluorescence. Gap junction intercellular communication (GJIC) was monitored using the scrape loading/dye transfer method.

Results: The results showed that treatment with testosterone at a physiological level significantly increased the PKC and p-Cx43 levels and GJIC activity in a concentration-dependent manner. However, the expression of PKC, p-Cx43 levels and GJIC activity in the cells treated with testosterone above physiological concentrations $\left(10^{-5} \mathrm{M}\right)$ decreased compared with those in the cells treated with $10^{-7} \mathrm{M}$ testosterone. Compared to the $10^{-7} \mathrm{M}$ testosterone-treated cells, the KGN cells treated with flutamide had much lower levels of PKC and p-Cx43. The levels of p-Cx43 and GJIC activity in the GF109203X-treated group were decreased compared to those in the $10^{-7} \mathrm{M}$ testosterone group. Immunofluorescence showed that testosterone treatment had no effect on the localization and distribution of gap junction proteins.

Conclusions: This study suggests that androgen can increase GJIC activity in granulosa cells by phosphorylating $\mathrm{Cx} 43$ via the PKC signalling pathway to promote oocyte development. The results of this study might provide a theoretical basis for androgen pretreatment of patients with POR.

\section{Background}

For controlled ovarian hyperstimulation $(\mathrm{COH})$, acquisition of the correct number of high-quality oocytes and production of multiple high-quality embryos are key steps to improving the clinical pregnancy rate. However, approximately $5.6-35.1 \%$ of women experience poor ovarian response (POR)[1], which is characterized by insensitivity to ovarian stimulation, fewer retrieved oocytes, an increased cycle cancellation rate, and reduced clinical pregnancy rates. Several stimulatory regimens and interventions have been proposed to improve POR. POR is common in patients with diminished ovarian reserve (DOR), while the latter is closely related to age. Androgen concentration in ovary decreases sharply as age advances, so we speculate that androgen level of POR patients is lower than normal level. In addition to oestrogen and progesterone, androgens have been shown to play a crucial role in follicular recruitment, growth and development in recent years. Given the importance of androgens, domestic and foreign 
reproductive centres have added androgen as an adjuvant to in vitro fertilization (IVF) protocols for patients with POR to increase both the quantity and quality of oocytes and embryos and improve pregnancy outcomes[2, 3]. Meta-analyses have also shown that pretreatment with androgens in patients with POR can significantly improve the clinical pregnancy rate and live birth rate[1, 4], but the specific mechanism underlying the effect of androgens is still unclear.

Oocytes are in an avascular microenvironment, and the substances and regulatory signals needed for growth rely on intercellular communication between oocytes and granulosa cells and between granulosa cells and theca cells. Gap junctions play an important role in maintaining the oocyte microenvironment and promoting the development of follicles[5]. Gap junctions formed by connexins (Cx) mediate cell-cell communication by electrical and chemical coupling. Studies show that many kinds of gap junction proteins are expressed in ovarian tissues, and Cx43 is the predominant connexin in granulosa cells. Oocyte growth and development are known to correlate with efficient gap junction intercellular communication (GJIC) among granulosa cells. Therefore, the growth and development of oocytes will be directly affected when the expression and function of gap junctions of granulosa cells are abnormal. GJIC can be either chronically regulated by changing the expression of connexins or quickly regulated by changing the conduction velocity of GJIC or the opening and closing of gap junctions. The phosphorylation state at the carboxyl terminus of connexin is an important factor affecting GJIC. Various protein kinases and protein phosphatases regulate GJIC by phosphorylation and dephosphorylation at different amino acid sites. GJIC can be promoted by proper phosphorylation of Cx43, while GJIC can be inhibited by improper phosphorylation or dephosphorylation. Protein kinase $C$ (PKC) plays an important role in the regulation of cell metabolism, growth, proliferation and differentiation and is widely distributed in mammalian organs, tissues and cells. PKC affects GJIC by phosphorylating serine 262 and 368 of Cx43[6].

Studies have shown that androgens can interact with androgen binding sites on the cell membrane through nongenetic pathways and cause L-type voltage-gated calcium channels to open mediated by $\mathrm{G}$ protein, sequentially causing extracellular calcium influx and thus leading to a rapid increase in the intracellular calcium ion concentration[7]. Calcium ions are a ubiquitous second messenger in cells, and increased intracellular calcium ion concentrations can activate calcium-sensitive molecules, including PKC and calmodulin, thereby activating signal transduction pathways and regulating transcription factors[8]. Therefore, we speculate that androgens can activate the PKC pathway, and then, PKC phosphorylates $\mathrm{Cx} 43$ and further regulates GJIC, which finally affects follicular growth and oocyte quality. To verify this hypothesis, we designed this study. Through an in-depth discussion of the mechanism by which androgen promotes follicular development, we not only elucidated the physiological role of androgen but also clarified its target of action. The results of this study might provide a theoretical basis for androgen pretreatment of patients with POR.

Ovarian granulosa cells play an important role in follicular development, maturation, and atresia[9]. Changes in the expression of connexin and GJIC in granulosa cells can affect oocyte growth and development. Human granulosa cells are mainly obtained at the time of oocyte retrieval, while the 
granulosa cells at this time have been transformed into luteinized granulosa cells. These cells are unable to passage and are thus difficult to obtain and maintain in the basic culture system. The granulosa-like tumour cell line KGN is considered a useful model for studying the regulation of steroidogenesis, cell growth, and apoptosis of human granulosa cells[10,11]. The choice of testosterone $(T)$ concentration in culture medium in vitro is mainly based on the physiological concentration of androgens in follicle fluid and cytotoxicity tests of granulosa cells in vitro as previously described[12]. We treated granulosa cells with $\mathrm{T}$ at a concentration equivalent to the physiological concentration in follicular fluid $\left(10^{-7} \mathrm{M}\right)$, a low concentration $\left(10^{-11} \mathrm{M}\right)$, and a high concentration $\left(10^{-5} \mathrm{M}\right)$. To assess whether the effect of androgen on connexin was mediated through the androgen receptor (AR) pathway, we cultured cells with $10^{-7} \mathrm{M} \mathrm{T}$ and $10^{-6} \mathrm{M}$ flutamide (AR antagonist). To study the involvement of androgen in the promotion of GJIC via the PKC signalling pathway, we used an inhibitor of PKC, GF109203X (bisindolylmaleimide 1), before exposure to $10^{-7} \mathrm{MT}$.

\section{Methods}

\section{Cell culture}

The human ovarian granulosa cell line KGN was obtained from the RIKEN BioResource Center. Cells were cultured in DMEM/F12 medium (Gibco, USA) containing 10\% foetal bovine serum (FBS) (HyClone, USA) and $1.0 \%$ penicillin/streptomycin (PS) (Gibco, USA). KGN cells were seeded in $60-\mathrm{mm}$ plates $\left(1 \times 10^{6}\right.$ cells/plates) and cultured at $37^{\circ} \mathrm{C}$ with $5 \% \mathrm{CO}_{2}$ until the cells adhered to the wall. Cultured cells were divided into six groups: (1) control group, in which granulosa cells were cultured in DMEM without any treatment for $24 \mathrm{~h}$; (2) $10^{-11} \mathrm{M} \mathrm{T}$ group, in which granulosa cells were cultured in DMEM with $10^{-11} \mathrm{M} \mathrm{T}$ (Sigma, USA) for $24 \mathrm{~h}$; (3) $10^{-7} \mathrm{M} \mathrm{T}$ group, in which granulosa cells were cultured in DMEM with $10^{-7} \mathrm{M}$ $\mathrm{T}$ for $24 \mathrm{~h}$; (4) $10^{-5} \mathrm{M}$ T group, in which granulosa cells were cultured in DMEM with $10^{-5} \mathrm{M} \mathrm{T}$ for $24 \mathrm{~h}$; (5) $10^{-7} \mathrm{M} \mathrm{T}+$ flutamide group, in which granulosa cells were cultured in DMEM with $10^{-7} \mathrm{M} \mathrm{T}$ for $24 \mathrm{~h}$ after the $10^{-6} \mathrm{M}$ flutamide (Sigma, USA) treatment for $1 \mathrm{~h}$; (6) $10^{-7} \mathrm{M} \mathrm{T}+\mathrm{GF} 109203 \mathrm{X}$ group, in which granulosa cells were cultured in DMEM with $10^{-7} \mathrm{M} \mathrm{T}$ for $24 \mathrm{~h}$ after the $10^{-6} \mathrm{M}$ GF109203X (MCE, USA) treatment for $1 \mathrm{~h}$. The follow-up experiment was performed immediately following the culture.

Western blot

After the medium was discarded, the cells were washed twice with phosphate-buffered saline (PBS). Cells were lysed for $20 \mathrm{~min}$ in RIPA buffer with protease inhibitors (100:1) and then centrifuged at $12000 \mathrm{r} / \mathrm{min}$ at $4^{\circ} \mathrm{C}$ for $5 \mathrm{~min}$. The supernatant was transferred to a new centrifuge tube for Western blot analysis. After determination of the protein content by the bicinchoninic acid (BCA) protein assay, $25 \mu \mathrm{g}$ proteins were separated by $10 \%$ SDS-PAGE and transferred onto nitrocellulose membranes. After the membranes were blocked with $5 \%$ skim milk for $1 \mathrm{~h}$, they were incubated with Cx43 antibody (1:1000, CST, USA), phosphorylated connexin 43 ( $\mathrm{p}$-Cx43) antibody (1:1000, CST, USA), and PKC antibody (1:1000, Abcam, UK) for $1 \mathrm{~h}$ at room temperature and overnight at $4^{\circ} \mathrm{C}$. After three washes in Tris-buffered saline 
(TBS)-0.1\% Tween 20, the membranes were incubated with goat anti-rabbit IgG and goat anti-mouse IgG conjugated with horseradish peroxidase (HRP) (1:5000, Proteintech Group, China) for 1 hour at room temperature. The membranes were washed again as described above, and the immunoreactivity was examined by an enhanced chemiluminescence (ECL) system. As a control for normalization, the membranes were reprobed for the internal control GAPDH (1:5000, Atagenix, China). Films were scanned, and the optical density of the bands was measured with AlphaEaseFC. The relative quantities of $\mathrm{Cx} 43, \mathrm{p}$ $\mathrm{Cx} 43$, and PKC were determined with reference to GAPDH. The final data are expressed as the mean of the results of three independent experiments performed at different time points.

\section{Immunofluorescence}

KGN cells were seeded on coverslips in 60-mm plates and cultured as described above. After three washes with PBS, the cells were fixed in $4 \%$ paraformaldehyde for $15 \mathrm{~min}$ and blocked with goat serum for $30 \mathrm{~min}$ at room temperature. After the supernatant was discarded, the cells were incubated overnight with Cx43 antibody (1:1000, CST, USA) or p-Cx43 antibody $\left(1: 1000, \mathrm{CST}\right.$, USA) at $4^{\circ} \mathrm{C}$. After three washes, the cells were incubated with goat anti-rabbit IgG secondary antibody (1:5000, Proteintech Group, China) for $1 \mathrm{~h}$ at room temperature. DAPI was dropped on coverslips, incubated for $5 \mathrm{~min}$ in the dark, and then viewed and imaged under a fluorescence microscope.

Scrape loading/dye transfer

GJIC was determined according to the Scrape loading/dye transfer method described previously[13-15], which used Lucifer yellow dye that can diffuse through gap junctions. KGN cells were cultured as described above, and after the medium was discarded, the cells were washed three times with preheated PBS at $37^{\circ} \mathrm{C}$. Two millilitres of $0.05 \%$ Lucifer yellow dye preheated at $37^{\circ} \mathrm{C}$ was added to the culture dishes. The cell layer was gently cut with a sterile scalpel and then incubated for 3 min under dark conditions. After incubation, the cells were washed with PBS three times, fixed with $4 \%$ paraformaldehyde, viewed and imaged immediately under a fluorescence microscope. GJIC activity was defined as the distance that the Lucifer yellow dye transferred from the scrape line. Values are expressed as a fraction compared to the control.

Statistical analysis

All analyses were performed with Software Package for Social Sciences (SPSS) version 10.0 for Windows. All data are expressed as the mean \pm standard deviation (SD). Comparisons among multiple samples were performed with variance analysis, and intergroup multiple comparisons were performed with Bonferroni tests. $\mathrm{P}<0.05$ was considered statistically significant.

\section{Results}

T induces phosphorylation of $\mathrm{Cx} 43$ 
GJIC is regulated by the phosphorylation state of gap junction proteins, and we first investigated the effect of T on the phosphorylation of $\mathrm{Cx} 43$ in KGN cells. Cells were treated with different concentrations of $\mathrm{T}$, and the $\mathrm{p}-\mathrm{C} \times 43$ expression levels were examined. The results showed that $\mathrm{T}$ at a physiological concentration $\left(T \leq 10^{-7} \mathrm{M}\right)$ significantly increased the $\mathrm{p}-\mathrm{C} x 43$ levels in a concentration-dependent manner. The expression of $\mathrm{p}-\mathrm{C} x 43$ after treatment with $10^{-11} \mathrm{M}$ and $10^{-7} \mathrm{M} \mathrm{T}$ was significantly higher than that of the control group. However, the expression of $\mathrm{p}-\mathrm{Cx} 43$ in the cells treated with $\mathrm{T}$ above physiological concentrations $\left(10^{-5} \mathrm{M}\right)$ decreased compared with that in the cells treated with $10^{-7} \mathrm{M} \mathrm{T}$ (Fig. 1).

T-induced $\mathrm{Cx} 43$ phosphorylation is mediated by the PKC signalling pathway

Various protein kinases and protein phosphatases regulate GJIC by phosphorylation and dephosphorylation of different amino acid sites of $\mathrm{Cx} 43$. To study the mechanism of T-induced $\mathrm{Cx} 43$ phosphorylation, we investigated the effect of T treatment on the expression of PKC in KGN cells. The results showed that $T$ at a physiological concentration $\left(T \leq 10^{-7} \mathrm{M}\right)$ significantly increased PKC levels in a concentration-dependent manner. The expression of PKC in the group treated with $10^{-7} \mathrm{~mol} / \mathrm{L} T$ was significantly higher than that in the control group. However, the expression of PKC in the cells treated with T above physiological concentrations $\left(10^{-5} \mathrm{M}\right)$ was decreased compared with that in the cells treated with $10^{-7} \mathrm{M} \mathrm{T}$ (Fig. 2). The effect of T on PKC expression was consistent with that on Cx43 phosphorylation.

When KGN cells were treated with an AR antagonist (flutamide), the results showed that compared to the $10^{-7} \mathrm{M}$ T-treated cells, the KGN cells treated with flutamide had much lower levels of PKC (Fig. 2) and pCx43 (Fig. 1).

To further verify that T-induced $\mathrm{Cx} 43$ phosphorylation is mediated by the $\mathrm{PKC}$ signalling pathway, we added an inhibitor of PKC, GF109203X, before exposure to $10^{-7} \mathrm{M} \mathrm{T}$. The results showed that the levels of $\mathrm{p}-\mathrm{C} \times 43$ in the GF109203X-treated group were downregulated compared to those in the $10^{-7} \mathrm{M} \mathrm{T}$ group (Fig. 1).

T has no effect on the localization of $\mathrm{Cx} 43$ and $\mathrm{p}-\mathrm{Cx} 43$

To study the effect of T on the localization of $\mathrm{Cx} 43$ and p-Cx43 in granulosa cells, we performed immunofluorescence staining of KGN cells. The results showed that $\mathrm{Cx} 43$ and $\mathrm{p}-\mathrm{C} \times 43$ displayed punctate to linear expression as green fluorescent plaques on the borders of granulosa cells in all groups, which is the typical appearance of aggregated $\mathrm{Cx} 43$ channels in gap junction plaques. The localization and distribution of $\mathrm{p}-\mathrm{C} \times 43$ was quite similar to that of total $\mathrm{Cx} 43$ (Fig. 3). T treatment had no effect on the localization and distribution of gap junction proteins.

T promotes GJIC activity via the PKC signalling pathway 
To investigate the effect of T on GJIC, we evaluated cellular GJIC using the scrape loading/dye transfer technique. The results showed that $T$ at a physiological concentration $\left(T \leq 10^{-7} \mathrm{M}\right)$ increased GJIC activity in a concentration-dependent manner. The GJIC activity after treatment with $10^{-11} \mathrm{~mol} / \mathrm{L}$ and $10^{-}$ $7 \mathrm{~mol} / \mathrm{L}$ T was significantly higher than that of the control group. However, the GJIC in the cells treated with $\mathrm{T}$ above the physiological concentration $\left(10^{-5} \mathrm{M}\right)$ was lower than that of the cells treated with $10^{-7}$ M T. Compared to the $10^{-7} \mathrm{M}$ T-treated cells, the KGN cells treated with GF109203X had much lower GJIC activity (Fig. 4).

\section{Discussion}

POR is one of the most challenging issues in assisted reproductive technology. A recent meta-analysis evaluated the effects of different adjuvant treatment strategies, including dehydroepiandrosterone (DHEA), letrozole, recombinant luteinizing hormone, recombinant human chorionic gonadotrophin, estradiol, clomiphene citrate, progesterone, growth hormone (GH) and coenzyme Q10 (CoQ10), on pregnancy outcomes in POR. The results showed that DHEA, CoQ10 and GH resulted in better clinical outcomes in terms of achieving pregnancy, and a lower dosage of gonadotrophin was required for ovulation induction[1]. What is the mechanism by which androgens promote follicle growth and development and improve the ovarian response?

To explore the mechanism of androgen action, we investigated the expression of PKC signalling pathwayrelated proteins, phosphorylation of Cx43, Cx43 localization and GJIC activity after androgen pretreatment. Our results showed that in after treatment with $\mathrm{T}$ at a physiological concentration, the expression of PKC and p-Cx43 and GJIC in granulosa cells were enhanced with increasing androgen concentrations. In the physiological range, androgen can activate the PKC pathway, PKC phosphorylates serine 262 and 368 of Cx43, and then, phosphorylated Cx43 further promotes GJIC activity, which further enhances the material and information communication between oocytes and surrounding somatic cells, improves the ovary microenvironment, promotes the growth and development of follicles and oocyte quality, and finally improves the outcome of pregnancy.

Our results demonstrated that the expression of PKC, the phosphorylation of Cx43 and GJIC activity in granulosa cells were decreased when the androgen concentration was higher than physiological concentration. In other words, androgen levels higher than physiological concentrations can reduce GJIC activity between follicles and stroma and result in follicular arrest and disturbed ovulation, which may also be the mechanism underlying the effect of high concentrations of androgen on the growth and development of follicles in patients with polycystic ovary syndrome[16, 17]. This finding suggests that when developing treatment plans for patients with POR, clinicians should clarify whether the androgen level in the patient is lower than the normal value. In patients with POR with low androgen levels, androgen pretreatment before IVF cycles can improve androgen levels, improve ovarian responses and increase the number of oocytes retrieved during the process of COS. However, patients with POR with normal androgen levels cannot benefit from androgen pretreatment before IVF cycles. 
Our results showed that $\mathrm{T}$ had no effect on the localization of $\mathrm{Cx} 43$ and $\mathrm{p}-\mathrm{C} \times 43$ in granulosa cells but affected the phosphorylation state of connexin, which was consistent with previous studies[18]. This finding indicates that androgen regulates GJIC by affecting the opening and closing of gap junction channels.

\section{Conclusions}

In conclusion, androgen can increase GJIC activity in granulosa cells by phosphorylating Cx43 via the PKC signalling pathway to promote oocyte development. Through an in-depth analysis of the mechanism by which androgen promotes follicular development, we not only helped elucidate the physiological role of androgen but also clarified its target of action. The results of this study might provide a theoretical basis for androgen pretreatment of patients with POR.

\section{List Of Abbreviations}

$A R$, androgen receptor

$\mathrm{COH}$, controlled ovarian hyperstimulation

CoQ10, coenzyme Q10

Cx, connexins

DHEA, dehydroepiandrosterone

DOR, diminished ovarian reserve

$\mathrm{GH}$, growth hormone

GJIC, gap junction intercellular communication

IVF, in vitro fertilization

PKC, Protein kinase C

POR, poor ovarian response

T, testosterone

\section{Declarations}

\section{Ethics approval and consent to participate}

Not applicable. 


\section{Consent for publication}

Not applicable.

\section{Availability of data and material}

The datasets used and/or analysed during the current study are available from the corresponding author on reasonable request.

\section{Competing interests}

The authors declare that they have no competing interests.

\section{Funding}

This study was supported by the National Natural Science Foundation of China (Grant No. 81801442).

\section{Authors' contributions}

$Y Z$ and $Y X$ contributed to experimental design, manuscript preparation and supervise the whole experimental process. YZ, YK and SW were involved in conducting experimental. JY conceived the data analysis and manuscript preparation. All authors contributed to the article and approved the submitted version.

\section{Acknowledgements}

We thank American Journal Experts (AJE) for English language editing. This manuscript was edited for English language by AJE.

\section{References}

1. Zhang Y, Zhang C, Shu J, Guo J, Chang HM, Leung P, et al. Adjuvant treatment strategies in ovarian stimulation for poor responders undergoing IVF: a systematic review and network meta-analysis. Hum Reprod Update. 2020; 26: 247-63.

2. Casson PR, Lindsay MS, Pisarska MD, Carson SA, Buster JE. Dehydroepiandrosterone supplementation augments ovarian stimulation in poor responders: a case series. Hum Reprod. 2000; 15: 2129-32.

3. Barad DH, Gleicher N. Increased oocyte production after treatment with dehydroepiandrosterone. Fertil Steril. 2005; 84: 756.

4. Jeve YB, Bhandari HM. Effective treatment protocol for poor ovarian response: A systematic review and meta-analysis. J Hum Reprod Sci. 2016; 9: 70-81.

5. Gilchrist RB. Recent insights into oocyte-follicle cell interactions provide opportunities for the development of new approaches to in vitro maturation. Reprod Fertil Dev. 2011; 23: 23-31. 
6. Scemes E. Nature of plasmalemmal functional "hemichannels". Biochim Biophys Acta. 2012; 1818: 1880-3.

7. Machelon V, Nome F, Tesarik J. Nongenomic effects of androstenedione on human granulosa luteinizing cells. J Clin Endocrinol Metab. 1998; 83: 263-9.

8. Mellstrom B, Naranjo JR. Mechanisms of Ca(2+)-dependent transcription. Curr Opin Neurobiol. 2001; 11: 312-9.

9. Dzafic E, Stimpfel M, Virant-Klun I. Plasticity of granulosa cells: on the crossroad of stemness and transdifferentiation potential. J Assist Reprod Genet. 2013; 30: 1255-61.

10. Havelock JC, Rainey WE, Carr BR. Ovarian granulosa cell lines. Mol Cell Endocrionl. 2004; 228: 67-78.

11. Nishi Y, Yanase T, Mu Y, Oba K, Ichino I, Saito M, et al. Establishment and characterization of a steroidogenic human granulosa-like tumor cell line, KGN, that expresses functional folliclestimulating hormone receptor. Endocrinology. 2001; 142: 437-45.

12. Zhang $Y$, Xu Y, Kuai Y, Wang S, Xue Q, Shang J. Effect of testosterone on the Connexin37 of sexual mature mouse cumulus oocyte complex. J Ovarian Res. 2016; 9: 82.

13. El-Fouly MH, Trosko JE, Chang CC. Scrape-loading and dye transfer. A rapid and simple technique to study gap junctional intercellular communication. EXP Cell Res. 1987; 168: 422-30.

14. Lee $\mathrm{CH}$, Chen IH, Lee $\mathrm{CR}$, Chi $\mathrm{CH}$, Tsai MC, Tsai JL, et al. Inhibition of gap junctional Intercellular communication in WB-F344 rat liver epithelial cells by triphenyltin chloride through MAPK and PI3kinase pathways. J Occup Med Toxicol. 2010; 5: 17.

15. Zhou S, Fang Z, Wang G, Wu S. Gap junctional intercellular communication dysfunction mediates the cognitive impairment induced by cerebral ischemia-reperfusion injury: PI3K/Akt pathway involved. Am J Transl Res. 2017; 9: 5442-51.

16. Shang K, Jia X, Qiao J, Kang J, Guan Y. Endometrial abnormality in women with polycystic ovary syndrome. Reprod Sci. 2012; 19: 674-83.

17. Walters KA. Role of androgens in normal and pathological ovarian function. Reproduction. 2015; 149: R193-218.

18. Talhouk R, Tarraf C, Kobrossy L, Shaito A, Bazzi S, Bazzoun D, et al. Modulation of Cx43 and Gap Junctional Intercellular Communication by Androstenedione in Rat Polycystic Ovary and Granulosa Cells in vitro. J Reprod Infertil. 2012; 13: 21-32.

\section{Figures}




\section{B}

$10^{-7} \mathrm{M} \mathrm{T}+10^{-7} \mathrm{M} \mathrm{T}+$

Control $10^{-11} \mathrm{M} \mathrm{T} \quad 10^{-7} \mathrm{M} \mathrm{T} \quad 10^{-5} \mathrm{M} \mathrm{T}$ Flutamide GF109203X

p-Cx43

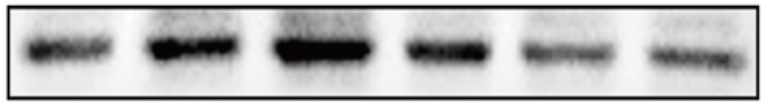

Cx43

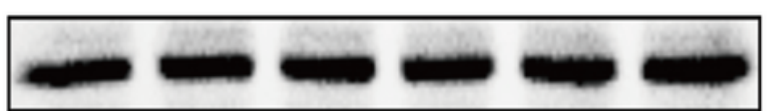

$44 \mathrm{KD}$

$43 \mathrm{KD}$

GAPDH

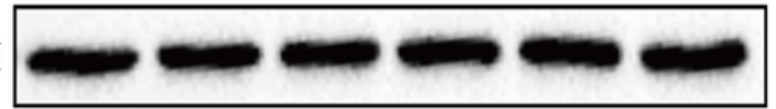

$36 \mathrm{KD}$

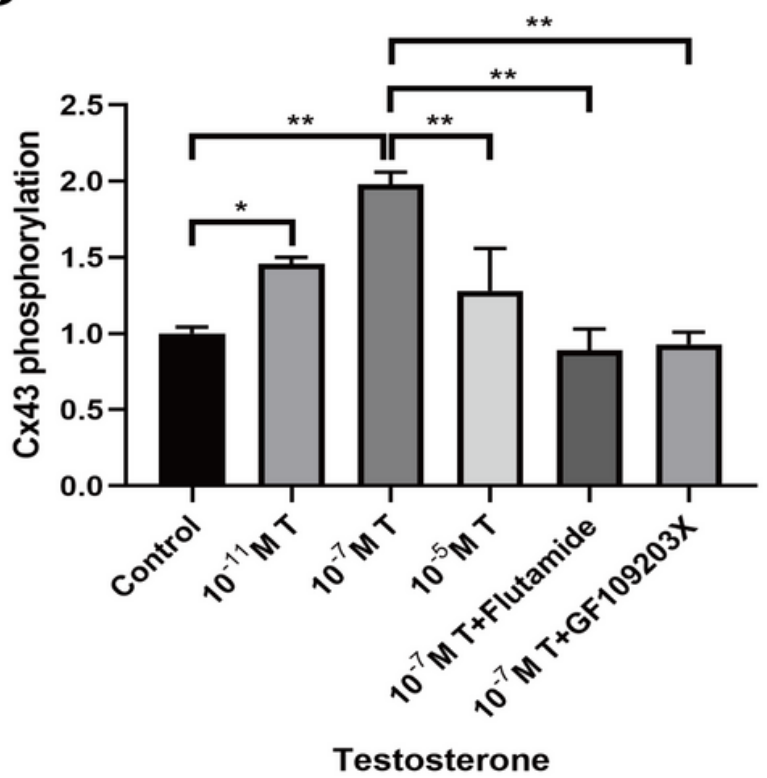

Testosterone

\section{Figure 1}

Effect of $\mathrm{T}$ on $\mathrm{Cx} 43$ phosphorylation by Western blot analysis. (A) $\mathrm{T}$ at a physiological concentration $\mathrm{T} \leq$ 10-7 M) significantly increased $\mathrm{p}-\mathrm{Cx} 43$ levels in a concentration-dependent manner. However, the expression of p-Cx43 after treatment with T above physiological concentrations decreased compared with that after treatment with 10-7 M T. The expression of p-Cx43 after treatment with 10-11 M and 10-7 M T was significantly higher than that of the control group. Compared to the 10-7 M T-treated cells, the KGN cells treated with flutamide and GF109203X had much lower levels of p-Cx43. (B) Relative expression of p-Cx43 protein in the control and T-treated KGN cells using Western blot analysis. The chart represents the intensity of $\mathrm{p}-\mathrm{C} \times 43$ proteins expressed as a relative optical density (ROD) in all groups. * represents $P<0.05$, ** represents $P<0.01$. 
A

$10^{-7} \mathrm{M} \mathrm{T}+$

Control $10^{-11} \mathrm{M} \mathrm{T} \quad 10^{-7} \mathrm{M} \mathrm{T} \quad 10^{-5} \mathrm{M} \mathrm{T}$ Flutamide

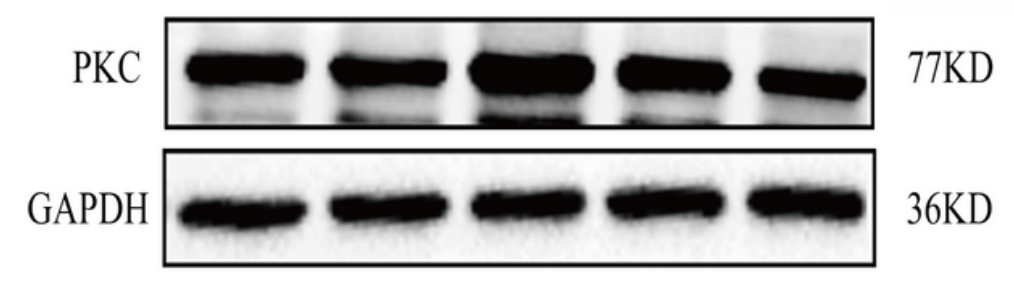

$\mathrm{B}$

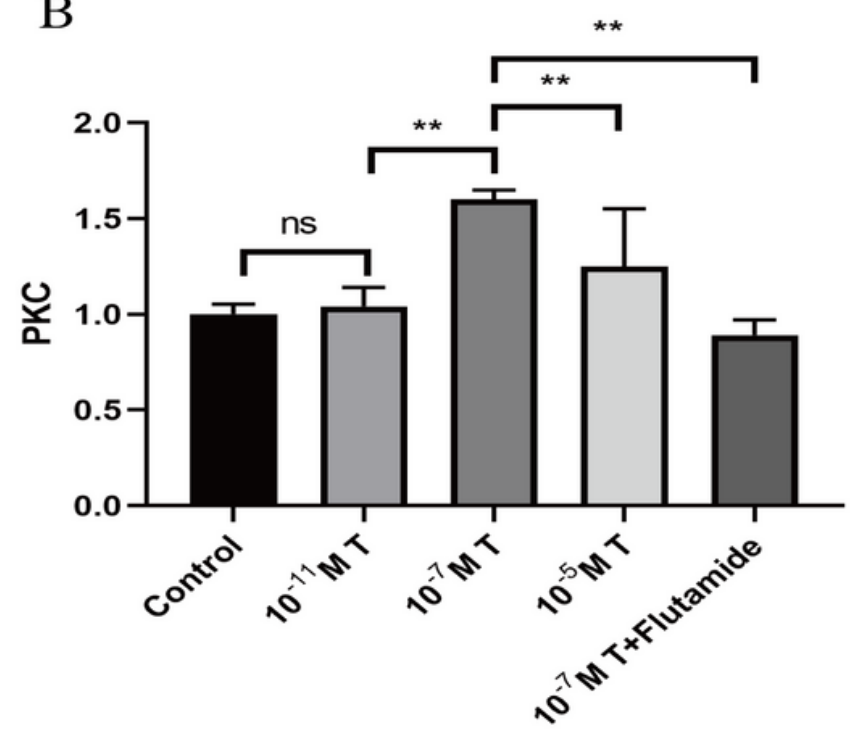

Testosterone

\section{Figure 2}

T-induced $\mathrm{C} x 43$ phosphorylation is mediated by the PKC signalling pathway. (A) The Western blot results showed that in $T$ at a physiological concentration $(T \leq 10-7 \mathrm{M})$ significantly increased PKC levels in a concentration-dependent manner. However, the expression of PKC in the cells treated with $T$ at physiological concentrations was decreased compared with that in the cells treated with 10-7 M T. Compared to the 10-7 M T-treated cells, the KGN cells treated with flutamide had much lower levels of PKC. (B) Relative expression of PKC proteins in the control and T-treated KGN cells using Western blot analysis. The chart represents the intensity of PKC proteins expressed as a ROD in all groups. ** represents $\mathrm{P}<0.01$. 
A

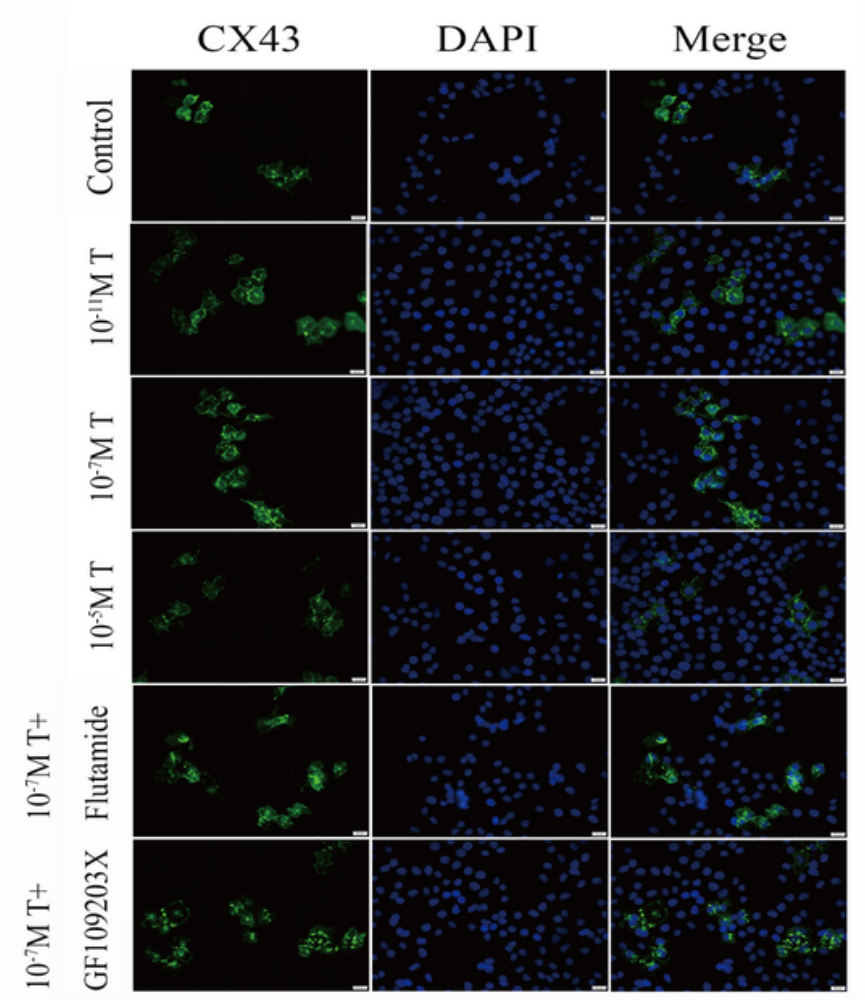

$\mathrm{B}$

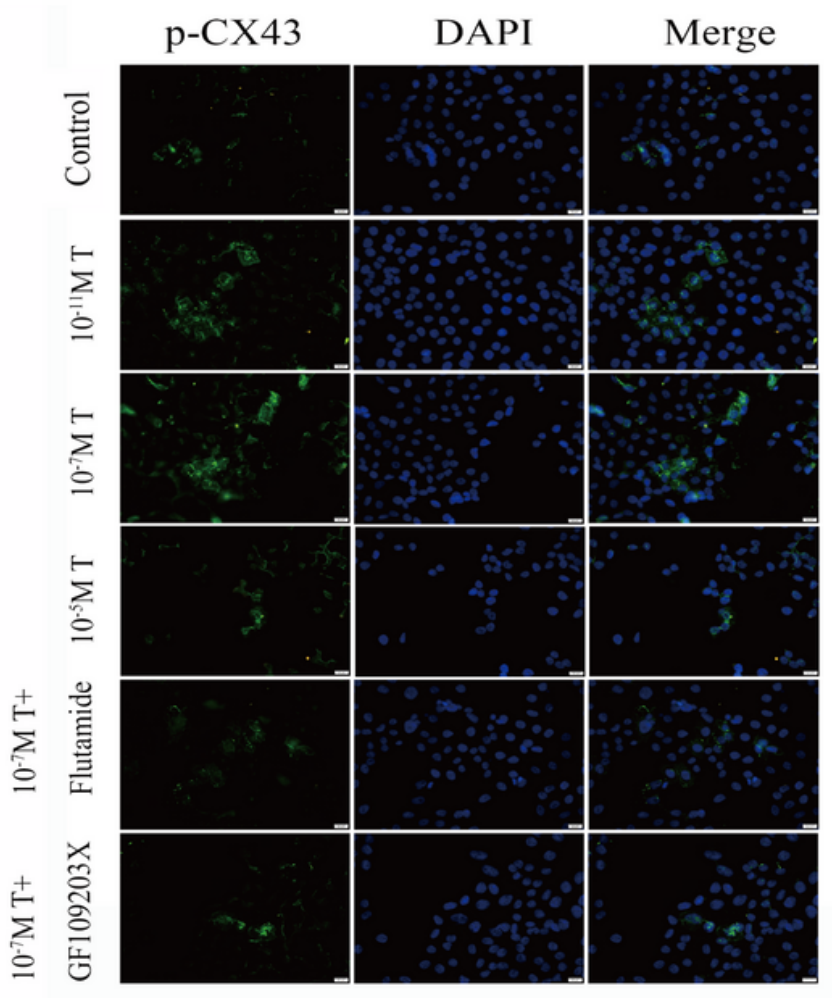

\section{Figure 3}

Effect of $\mathrm{T}$ on the localization of $\mathrm{Cx} 43$ and p-Cx43 by immunofluorescence. Staining of $\mathrm{Cx} 43(\mathrm{~A})$ and $\mathrm{p}$ Cx43 (B) in the control cells or in the cells treated with 10-11 M T, 10-7 M T, 10-5 M T, 10-7 M T + flutamide, and 10-7 M T + GF109203X. Cx43 and p-Cx43 displayed punctate to linear expression on the borders of granulosa cells in all groups. $T$ treatment had no effect on the localization and distribution of gap junction proteins. 
A

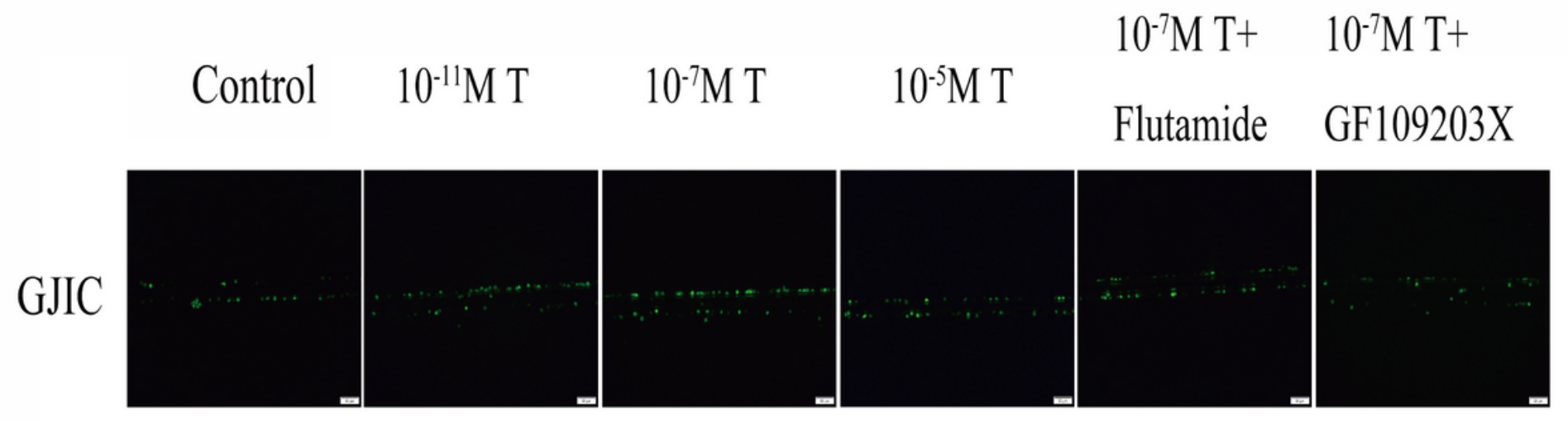

$\mathrm{B}$

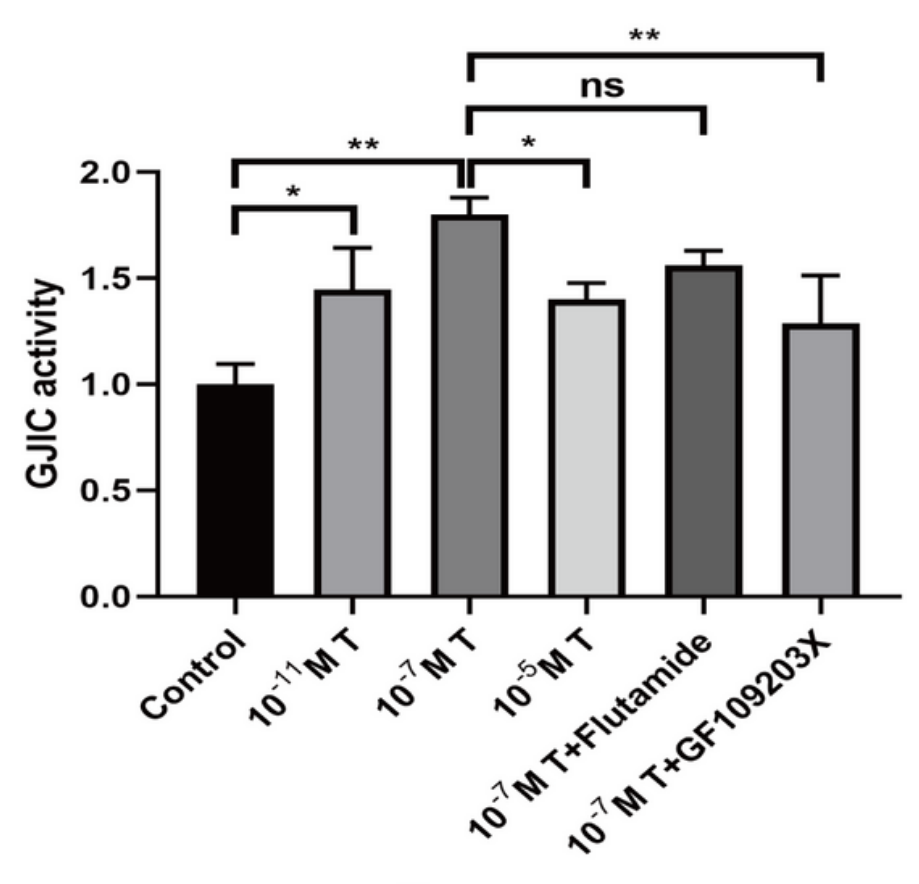

Testosterone

\section{Figure 4}

T promotes GJIC through the PKC signalling pathway. (A) The results of the scrape loading/dye transfer technique showed that $T$ at a physiological concentration increased GJIC activity in a concentrationdependent manner. However, the GJIC of the cells treated with T above physiological concentrations was lower than that of the cells treated with 10-7 M T. Compared to the 10-7 M T-treated cells, the KGN cells treated with flutamide and GF109203X had much lower GJIC activity. (B) Quantitative analyses of relative dye transfers (representative of GJIC activity). * represents $P<0.05$, ** represents $P<0.01$. 